\title{
Machine Learning Based Analysis of the Effects of Image Transformation and Partial Information on Face Recognition with Time-Varying Expressions for Homeland Security Applications
}

\author{
Dalila B. Megherbi \\ Computer Machine/Human Intelligence Networking and Distributed Systems (CMINDS) Research Center \\ Faculty of Electrical and Computer Engineering, Francis College of Engineering \\ University of Massachusetts Lowell
}

USA

Many machine learning and facial recognition algorithms display complex performances, yet little is known about these performance complexities. Current deep learning techniques for machine vision are limited. Adding different layers and increasing the size of training data (big data) will not be enough to shed light on these learning complexities. Facial recognition, especially with time-varying facial expressions and/or disguises, is crucial in many homeland security applications. The re-occurring terrorist attacks are reminder examples of the importance of developing accurate and reliable facial recognition algorithms. While various face recognition algorithms have been proposed in the literature, unfortunately many of them still remain in their infancy. This is mainly due to their lack of high recognition rates in the presence of varying image face artifacts and conditions, even slight ones. In order to develop more accurate facial recognition systems, there is a primary need to identify and derive some of the causes that affect some face recognition accuracy rates. In this talk I will focus and present our latest findings on investigation and analysis of how and what factors, including incomplete facial information, may affect the recognition accuracy rate of some of the most popular and currently widely used face recognition algorithms. Detection of wanted or suspicious individuals encountered generally at airports or transportation stations, via the recognition of their faces for the purpose of security, is an important area for preventing terrorism and smuggling. Due to rapid increase in the size of digital image collections and the difficulties encountered in automatic image annotation and/or manually-generated text-based image retrieval, I will also discuss some content-based image retrieval techniques in a distributed computing setting. 